\title{
Analyse génétique du comportement en open-field du jeune poussin (Gallus gallus domesticus)(1)
}

\author{
J.M. FAURE \\ I.N.R.A., Station de Recherches avicoles, \\ Centre de Recherches de Tours-Nouzilly \\ F 37380 Monnaie
}

\begin{abstract}
Résumé
Le test d'open-field a été très utilisé chez les rongeurs de laboratoire mais beaucoup moins pour les oiseaux. Il présente l'avantage d'être très standardisé et rapide et s'applique donc bien aux études de génétique.
\end{abstract}

\section{I. - Etude du comportement en open-field}

L'open-field utilisé permet l'enregistrement automatique de la latence et de l'activité de l'animal. Il s'agit d'une enceinte circulaire de $1 \mathrm{~m}$ de diamètre divisée en 6 secteurs par 6 rayons lumineux aboutissant chacun à une cellule photo-électrique. Les cris, les déjections et les sauts sont mesurés par l'observateur.

L'étude d'un échantillon de souches nous a permis de montrer que les différentes mesures sont corrélées entre elles (corrélations négatives entre la latence et les autres caractères, positives entre tous les autres caractères) et qu'il n'y a pas de différences dues au sexe. De plus, ce test est peu sensible aux conditions de milieu.

\section{II. - Etude génétique}

Après huit générations de sélection, les lignées sont très différentes et les distributions ne se recouvrent pratiquement pas. Les héritabilités des différents caractères présentent des valeurs faibles ou moyennes. Les caractères étudiés ne présentent pas d'hétérosis ou d'homéostase de développement. Pour plusieurs caractères, on constate la présence d'un effet maternel allant dans le sens d'une dissemblance entre la mère et ses descendants.

Cet ensemble de caractères permet de déduire que la motivation sous-tendant le comportement en open-field a été soumise à une sélection naturelle stabilisante.

\section{III. - Caractères évoluant corrélativement à la sélection}

Les animaux de la lignée active sont caractérisés par une émotivité plus faible et un taux de corticostérone réduit, ils sont aussi moins agressifs. Les animaux ayant une activité en open-field moyenne ont une aptitude à la dominance plus élevée.

Les lignées présentent au contraire peu de différences pour les caractères d'intérêt zootechnique.

(1) Résumé de thèse de doctorat ès Sciences présentée à l'Université de Toulouse ( $\mathrm{n}^{\circ}$ d'ordre 1010) le 29-9-1981. 
Un modèle est proposé pour essayer d'expliquer le comportement en open-field du jeune poussin en fonction de l'émotivité et de la motivation sociale.

Les animaux actifs peu émotifs, peu agressifs et peu sensibles aux agents stressants semblent correspondre à un animal plus adaptable aux conditions d'élevage moderne et ce type de sélection facilement utilisable sur des grands nombres d'animaux pourrait donc être utilisé en sélection commerciale.

\section{Introduction}

La connaissance empirique du comportement animal est probablement aussi vieille que l'humanité, dans la mesure où seule cette connaissance a pu permettre la chasse puis la domestication des animaux. Cependant, cette connaissance est restée au stade empirique jusqu'à la fin du siècle dernier.

De même, dès le début de la domestication, il y a eu sélection des races spécialisées pour certains types de comportements (Wood-GusH, 1959 ; Fuller, Du BuIs, 1969) : coqs de combat, races spécialisées de chiens, alors que le développement de la génétique du comportement ne date que d'une vingtaine d'années.

L'apparition de problèmes d'adaptation au milieu d'élevage dans les espèces domestiques est un phénomène récent dû au décalage croissant entre les vitesses d'évolution des méthodes d'élevage et les possibilités d'évolution «naturelle » des espèces domestiques. Pour cette raison, les problèmes d'adaptation sont ceux qui sont le plus souvent rencontrés au cours du développement d'élevages de type industriel et ils présentent d'autre part une importance théorique très grande puisqu'ils sont $\mathrm{au}$ cœur du problème de l'évolution des espèces.

Ils peuvent être résolus soit par l'adaptation du milieu d'élevage à l'animal soit par l'adaptation de l'animal à son milieu (FAURE, 1980 a).

L'adaptation du milieu d'élevage à l'animal a été la solution utilisée jusqu'à une date récente mais les pressions économiques ont entraîné son abandon.

L'adaptation de l'animal au milieu d'élevage peut être réalisée par trois voies : la voie pharmacologique, la voie ontogénétique et la voie génétique.

La voie pharmacologique pose le problème du coût des drogues utilisées et aussi le problème des résidus; de plus, comme pour la voie ontogénétique, l'effort doit être recommencé à chaque génération.

La voie génétique permet au contraire d'obtenir un changement définitif en n'agissant qu'au niveau des reproducteurs donc sur un nombre relativement limité d'animaux. Elle présente par contre l'inconvénient de n'être efficace qu'après plusieurs années.

Pour cette dernière raison, il est donc important de ne sélectionner que pour des caractères qui sont exprimés quelles que soient les conditions de milieu puisque dans le cas contraire, on prend le risque qu'une évolution du milieu rende inefficace l'effort entrepris.

La sélection est la méthode qui seule peut être utilisée sur le plan pratique pour entraîner une évolution du comportement. De plus, l'utilisation de lignées divergentes permet d'étudier au mieux l'évolution des caractères corrélés au caractère sélectionné. 
Le comportement en open-field a été choisi, car il permet, d'une part de mesurer l'adaptabilité des animaux et d'autre part, du point de vue pratique la brièveté des tests fait qu'ils sont particulièrement bien adaptés à l'analyse génétique qui nécessite la mesure de nombre élevé d'animaux (FAURE, 1981 a).

Le comportement en open-field des oiseaux ayant été peu étudié, nous avons dans une première partie analysé ce comportement chez le jeune poussin.

Dans une seconde partie, nous avons abordé les aspects génétiques de ce comportement chez la poule puis dans une troisième partie, nous verrons comment d'autres caractères de comportement ou d'intérêt économique ont évolué au cours de la sélection.

\section{Matériel et méthodes}

\section{1. - Méthode de mesure}

L'open-field utilisé (FAURE, Folmer, 1975) est représenté par la figure 1.

Il est composé d'une cuve cylindrique de $1 \mathrm{~m}$ de diamètre et de $70 \mathrm{~cm}$ de haut. Les parois sont peintes en noir mat. Le fond est recouvert d'une couche de copeaux de bois.
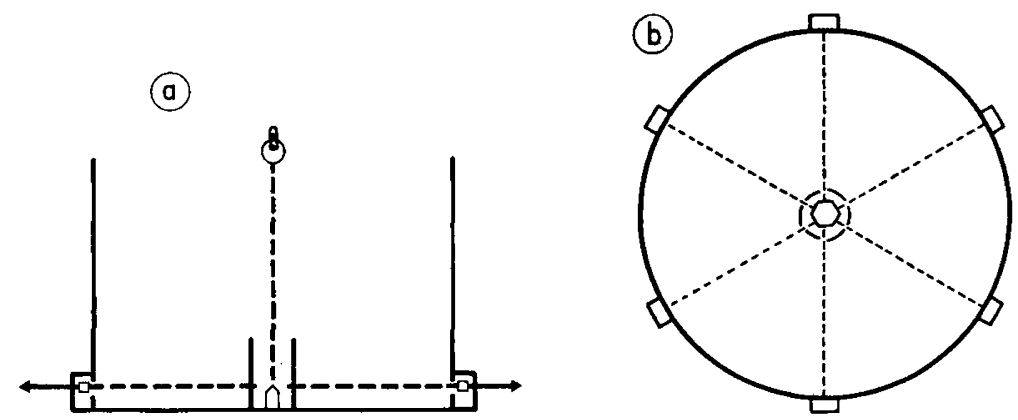

FIG. 1

Vue de l'open-field en coupe $(1$ a) et en plan $(1 \mathrm{~b})$.

On voit au centre de la cuve le prisme à 6 faces et dans la paroi les cellules photo-électriques.

Les pointillés indiquent le trajet des rayons lumineux.

Plan and elevation diagram of open-field apparatus.

A hexagonal prism is situated in the center and photo-electric cells are incorporated in the walls.

Le centre de la cuve est occupé par un prisme hexagonal dont les faces inclinées à $45^{\circ}$ renvoient la lumière produite par une lampe infra-rouge de 250 watts sur 6 photo-transistors. Cette lampe sert simultanément à l'éclairage et au chauffage des poussins. La latence, le nombre total de rayons lumineux coupés et le nombre de rayons lumineux différents coupés par les poussins sont enregistrés automatiquement. 
Les animaux sont testés à l'âge de 2 ou 3 jours. Ils sont mis la veille du test dans une cage placée dans une pièce contiguë à la salle où se trouve l'open-field. Ils sont chauffés, nourris et abreuvés. L'animal à tester est placé entre deux cellules et l'interrupteur mis sur la position départ.

Si l'animal ne coupe pas un des faisceaux lumineux avant la fin d'une période fixée à 100 secondes, il est noté latence $=100$ secondes et activité $=0$. Si l'animal coupe un faisceau lumineux avant la fin de la période de 100 secondes, un compteur affiche la valeur de cette latence alors que 6 autres compteurs mesurent le nombre d'interruptions de chacun des faisceaux lumineux pendant une autre période de 100 secondes.

Les caractères mesurés automatiquement sont donc :

- la latence, c'est-à-dire le temps en seconde que met le poussin pour couper le premier faisceau lumineux ;

- l'activité mesurée par le nombre total de faisceaux lumineux interrompus;

- le nombre de cellules, égal au nombre de faisceaux lumineux différents qui sont coupés.

En plus de ces caractères, un observateur note :

- les cris par une note à deux niveaux suivant que le poussin émet le « cri de détresse » pendant plus ou moins de 80 des 100 premières secondes;

- la présence ou l'absence de défécation pendant les 100 premières secondes. Chez le poussin, il est sans intérêt de compter les défécations puisque moins de 1 p. 100 des animaux testés défèquent plus d'une fois;

- le nombre de sauts contre les parois de l'open-field.

\section{2. - Validité des mesures}

Deux types de causes d'invalidité des tests peuvent se présenter :

- une variation des résultats en fonction du temps;

- une relation faible entre les mesures obtenues par l'open-field automatique et le trajet effectivement parcouru.

Les variations en fonction du temps pourraient être dues à des différences entre les poussins testés à l'âge de 2 jours et ceux testés à l'âge de 3 jours, à un rythme circadien, ou à un rythme infracircadian (GuYOMARC'H, 1975). Aucun de ces effets n'est significatif. Nous avons aussi calculé la corrélation entre l'activité et la latence mesurée par l'appareil automatique et le nombre de pas d'une part, et la latence mesurée par un observateur d'autre part (FAURE, JonES, 1981).

La corrélation est très élevée entre l'activité et le nombre de pas ( $r=0,94)$. Elles est par contre relativement faible entre les deux mesures de la latence $(r=0,55)$.

Ceci est dû à deux causes :

- certains animaux n'effectuent que quelques pas au cours du test et peuvent donc ne pas couper de rayon lumineux tout en ayant une latence courte quand elle est mesurée par l'observateur ;

- la course panique n'est pas prise en compte par l'observateur. 
Il faut remarquer qu'une mesure classique de la validité : la répétabilité ne peut pas être appliquée au présent test puisque le comportement en open-field est une réaction à un milieu nouveau.

Il n'y a pas non plus de différences dues au sexe dans le comportement en open-field et dans les conditions de tests utilisées (FAURE, $1979 \mathrm{~b}$; Jones \& FaURE, 1981, 1982). Ceci nous permettra par la suite de toujours traiter ensemble les mâles et les femelles.

\section{3. - Méthode de sélection et estimation des paramètres génétiques}

Le caractère sélectionné est le produit de l'activité par le nombre de cellules ceci afin d'éliminer de la lignée active les animaux qui marquent une activité élevée en n'utilisant qu'une cellule et donc en ne se déplaçant que très peu.

L'étude génétique a été effectuée sur une souche de type Cornish et a été conduite pendant 8 générations (FaURE \& Folmer, 1975 ; FaURE, 1981 b).

Les reproducteurs (10 mâles et 50 femelles par lignée) ont été choisis sur leur valeur génétique calculée en tenant compte à la fois du phénotype de l'individu et de la moyenne de la frâtrie. Il s'agit donc d'une sélection combinée (BoYER, 1958; FALCONER, 1961).

Les héritabilités calculées l'ont été à partir de l'analyse de variance hiérarchique. Les valeurs indiquées sont des moyennes de la composante père et de la composante mère.

Le calcul des héritabilités réalisées a été fait par la méthode décrite par FALCONER (1961).

\section{4. - Réalisation des croisements}

Un croisement mendelien complet avec production des 2 F 1 réciproques, des $4 \mathrm{~F} 1$ et des 8 back-cross possibles a été réalisé à partir des animaux de la $6^{\mathrm{e}}$ génération.

Les caractères mesurés sont la latence, la latence des «cris de détresse », l'activité, le nombre de cellules utilisées, le nombre de sauts et la présence ou l'absence de défécation. Les mêmes mesures ont été prises pendant 4 répétitions du test aux âges de $2,3,4$ et 5 jours. Le caractère sélectionné (produit de l'activité par le nombre de cellules) n'a pas été utilisé dans cette expérience car s'il est intéressant au niveau individuel, il est très comparable à l'activité au niveau des moyennes.

Pour la production des back-cross, nous avons utilisé soit des mâles F 1 et des femelles parentales vieilles, soit des mâles des lignées parentales et des femelles F 1 jeunes. Ces femelles jeunes ont des œufs et des poussins plus petits, il nous a donc été possible de comparer des animaux de génotypes semblables mais de taille différente.

Aucun des caractères mesurés ne satisfait à la fois les tests de Cochran d'homogénéité des variances dans les lignées parentales et les F 1 et les tests de MATHER, et ceci même après transformation des données (MATHER, 1949; BROADHURST \& JiNKS, 1961). 


\section{5. - Caractères évoluant corrélativement à la sélection}

Un certain nombre de caractères non directement liés au caractère sélectionné ont été mesurés au cours de la sélection afin de contrôler leur évolution. Il s'agit soit de caractères de comportement (réactivité émotionnelle, comportement social...) ou de caractères d'intérêt zootechnique (conformation, reproduction...).

\section{Résultats}

\section{1. - Etude descriptive du comportement en open-field}

\section{a) Description du comportement en open-field}

Cette description est résumée dans la figure 2.

Quand il est posé dans l'open-field, le poussin est d'abord immobile et silencieux dans la majorité des cas. Il peut adopter une position debout, assise ou couchée.

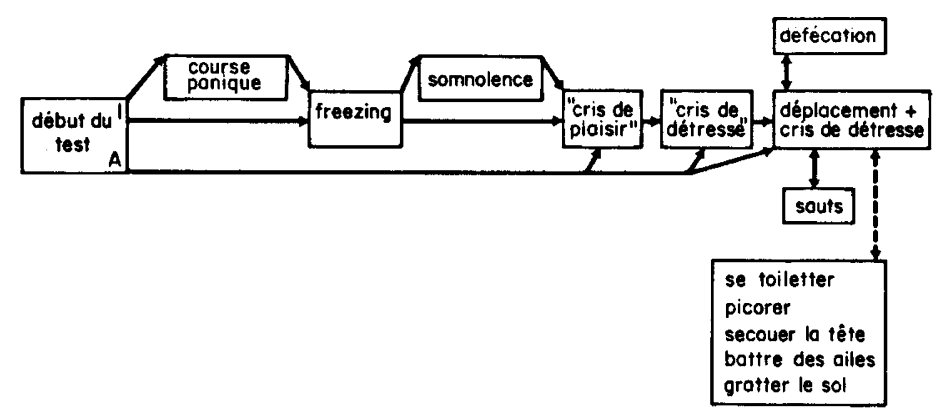

FIG. 2

Organisation du comportement en open-field du jeune poussin.

Les flèches indiquent dans quel sens s'organise la séquence.

Les flèches épaisses indiquent la succession d'actes les plus probables, les flèches en pointillé les séquences très rares.

Dans la case début du test, le I indique la séquence la plus fréquemment suivie par les inactifs,

le $A$ indique celle qui est la plus fréquente pour les actifs.

The sequence of behaviour patterns shown by young domestic chicks in the open-field.

Heavy lines indicate the commonest sequence of behaviour patterns

and the dotted line refers to those behaviour patterns which occur relatively rarely.

Le poussin présente souvent au début du test une période de «freezing 》 caractérisée par une immobilité complète et l'absence de cris.

Cette première phase peut être suivie par :

- une phase de somnolence,

- par une période d'émission de cris, qui sont d'abord du type «cris de plaisir » et qui sont progressivement remplacés par des «cris de détresse ». 
Cette période correspond aussi à une phase d'observation de l'environnement.

Ce n'est qu'après l'émission des «cris de détresse » que le poussin commence à se déplacer, et cette émission continue pendant la phase de déplacement.

C'est pendant cette phase de déplacement qu'il peut y avoir des défécations, des sauts ou exceptionnellement des activités de type confort (toilettes, picorer, se secouer, battre des ailes, gratter le sol...), ces dernières activités n'ayant pas été notées systématiquement car elles ne sont réalisées que par moins de 2 p. 100 des animaux.

Une variante fréquemment observée est un départ rapide du poussin au moment où il est posé dans l'open-field. Il parcourt ainsi quelques dizaines de centimètres, puis s'arrête et reprend au début la succession des comportements précédemment décrits.
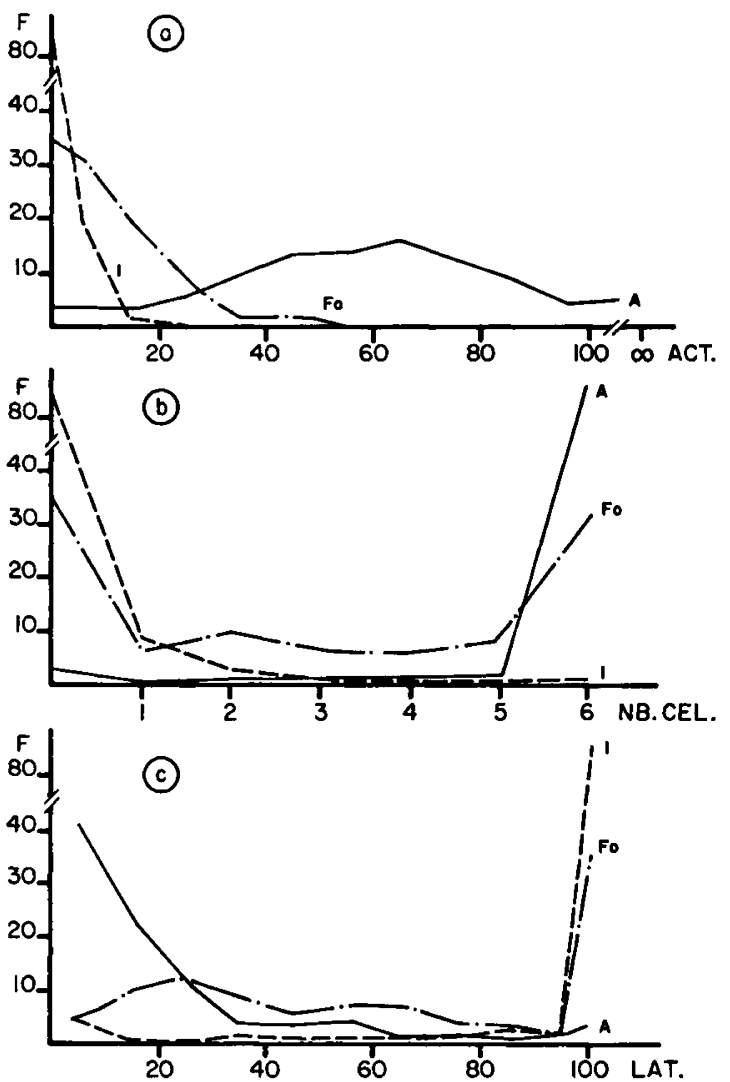

FIG. 3

Distribution de l'activité ( $3 a$ ), du nombre de cellules $(3 b)$ et de la latence $(3 c)$ pour les FO (tirets longs), les animaux actifs (trait plein) et inactifs (tirets courts) de la huitième génération.

Distribution of activity (a), number of cells (b) and latencies (c) of the starter pool (dashes), in the eighth generation of the active strain (solid line) and in the eighth generation of the inactive strain (dots). 
Toutes les phases, à l'exception de la course panique qui est toujours brève, ont une durée qui peut varier de 0 seconde à une valeur bien supérieure à la durée du test. La fin du test peut donc intervenir au cours de chacune des phases.

Les possibilités de variation de la séquence sont très limitées, sinon par suppression de certaines activités par rapport à une séquence théorique complète.

\section{b) Distribution des mesures}

La figure 3 montre pour la souche de départ la distribution de l'activité du nombre de cellules et de la latence. On constate que les distributions de l'activité et de la latence sont des courbes en $i$ avec l'une des classes extrêmes contenant 30 p. 100 des animaux. Pour le nombre de cellules, la distribution présente une forme en $U$, les deux classes extrêmes étant les plus fréquentes.

Ce type de distribution pose un problème pour l'analyse génétique puisque aucune des transformations simples ne permet de les normaliser.

Les tests statistiques courants nécessitant la normalisation des données, nous avons utilisé la transformation probit (Cochez, Pero, 1954). En effet, cette méthode s'applique particulièrement bien à un caractère à seuil et oblige seulement à supposer que le caractère mesuré repose sur une variable sous-jacente normalement distribuée, mais n'entraîne pas d'autres hypothèses quant aux propriétés de la distribution observée.

\section{TABLEAU 1}

Signe des corrélations entre les différents caractères mesurés en open-field.

Sign of the correlations between various characters measured in open-field.

\begin{tabular}{|c|c|c|c|c|c|c|}
\hline & Lat. & Act. & Nb. cel. & Cr. & Déj. & S. \\
\hline 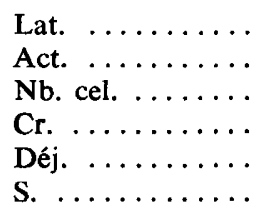 & & 一 & $\overline{+}$ & $\begin{array}{l}\overrightarrow{+} \\
+\end{array}$ & $\begin{array}{c}0- \\
+ \\
+ \\
+\end{array}$ & $\begin{array}{l}+ \\
+ \\
+ \\
+\end{array}$ \\
\hline
\end{tabular}

$\begin{array}{cl}\text { Lat. } & : \begin{array}{l}\text { Latence. } \\ \text { Latency. }\end{array} \\ \text { Act. } & : \begin{array}{l}\text { Activité. } \\ \text { Activity. }\end{array} \\ \text { Nb. cel. } & : \begin{array}{l}\text { Nombre de cellules utilisées. } \\ \text { Number of cells. }\end{array} \\ \text { Cr. } & : \begin{array}{l}\text { Cris. } \\ \text { Distress calls. }\end{array} \\ \text { Déj. } & : \begin{array}{l}\text { Déjections. } \\ \text { Defecations. }\end{array} \\ \text { S. } & : \begin{array}{l}\text { Sauts. } \\ \text { Jumps. }\end{array}\end{array}$




\section{c) Liaisons entre les différents caractères}

Le signe de ces liaisons est résumé dans le tableau 1. Il s'agit d'une présentation synthétique des corrélations intra-souches et des corrélations entre les valeurs moyennes d'un échantillon de 12 souches. On constate que l'ensemble des caractères peut se réduire à deux groupes : la latence d'une part, qui est corrélée négativement avec tous les autres caractères, les autres caractères d'autre part, qui sont tous positivement corrélés entre eux.

Dans cette première partie, nous avons vu que, dans les limites utilisées, le comportement en open-field ne varie pas en fonction de l'âge, n'est pas sensible aux rythmes courts d'activité ou à l'heure du test et ne présente pas de différences dues au sexe. Ces trois caractéristiques permettent donc de comparer deux animaux quel que soit le jour ou l'heure de la mesure et quel que soit leur sexe. C'est évidemment un avantage important pour la sélection.

L'étude des liaisons entre les différents paramètres mesurés suggère que l'on peut classer les animaux en deux catégories :

- les poussins actifs présentant donc une activité élevée, mais aussi une latence courte, une fréquence élevée des cris, des sauts et des défécations;

- les poussins inactifs ayant les caractéristiques inverses.

Cette dichotomie est particulièrement favorable à la réalisation d'une sélection divergente.

\section{2. - Etude génétique}

a) Résultats de la sélection

\section{- Distribution des mesures :}

La figure 3 représente les distributions de l'activité, du nombre de cellules et de la latence pour la génération de départ (tirets longs) et les lignées actives (traits pleins) et inactives (tirets courts) après 8 générations de sélection.

Pour l'activité, on constate que la forme en $i$ de la distribution dans la génération 0 a été exagérée par la sélection dans la lignée inactive (75 p. 100 des animaux ont une activité nulle) alors que dans la lignée active, la distribution se rapproche d'une distribution normale. Le recouvrement entre les deux distributions est très faible puisque aucun des inactifs n'a une activité supérieure à 20 alors que seulement 10 p. 100 des actifs ont un score inférieur à cette valeur.

La distribution du nombre de cellules qui présentait une forme en $U$ dans la population de départ a été transformée en une distribution en $J$ dans la lignée active, avec 90 p. 100 des animaux utilisant 6 cellules et en une distribution en $i$ dans la lignée inactive avec 85 p. 100 des animaux ne se déplaçant pas. Pour ce caractère aussi le recouvrement de distributions des deux lignées est faible.

A la génération 0 , la latence présentait une distribution complexe avec un premier mode entre 20 et 30 secondes et 35 p. 100 des animaux ayant une latence de 100 secondes. Dans la lignée inactive, le premier mode disparaît et la proportion d'oiseaux ayant une latence de 100 secondes passe à 85 p. 100. Dans la lignée active, cette proportion est réduite à 3 p. 100 alors que la fréquence des latences inférieures 
à 30 secondes passe de 27 p. 100 à la génération 0 à 78 p. 100 pour la $8^{e}$ génération.

- Evolution des mesures au cours de la sélection :

On constate (fig. 4) que le produit, qui est le caractère sélectionné, et l'activité présentent des évolutions très comparables.

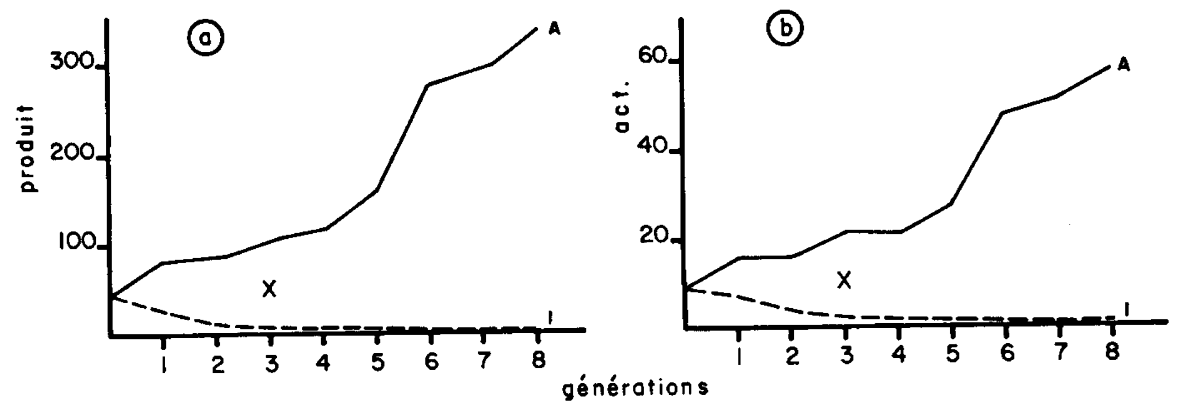

FIG. 4

Evolution, au cours de la sélection, du caractère sélectionné

(produit de l'activité et du nombre de cellules utilisées) (4a) et de l'activité (4 b) (actifs : trait plein, inactifs : tirets, $X$ : témoins mesurés avec les animaux de la troisième génération).

Changes in the selected characters as a function of the generation for the product (a) and the activity $(b)$. Active strain : solid line; inactive strain : dotted line. $X$ shows the mean of unselected strain measured with the birds of the third generation.

L'évolution est à peu près symétrique jusqu'à la $3^{\text {e }}$ génération puis présente un plateau dû à la proximité de la limite 0 dans la lignée inactive alors que dans la lignée active, il n'y a pas de signe de plateau même après 8 générations de sélection.

La comparaison des distributions en pourcentage cumulé aux générations 0 , 4 et 8 en tenant compte des animaux ne se déplaçant pas (fig. 5 a) et sans en tenir compte (fig. $5 \mathrm{~b}$ ) montre que dans la lignée active la sélection a porté aussi bien sur la proportion d'oiseaux ne se déplaçant pas que sur l'activité moyenne de ceux qui se déplacent. Il en a bien été de même dans la lignée inactive jusqu'à la $4^{\mathrm{e}}$ génération, mais ensuite, seule la proportion d'animaux ne se déplaçant pas a varié.

Globalement, l'évolution du nombre de cellules est symétrique (fig. 6 a) on constate cependant que dans la lignée inactive cette évolution est régulière alors que dans la lignée active, il y a des phases d'accroissement rapide (générations 0 à 1 ou 4 à 6) entre-coupées de palliers (générations 1 à 4 ou 6 à 8).

Une explication possible de ce résultat consisterait à supposer qu'à la $4^{e}$ génération il $\mathrm{y}$ a eu recombinaison entre les gènes majorants et minorants précédemment liés.

Pour la latence (fig. 6 b), l'évolution globale est à peu près régulière, mais la courbe de distribution en pourcentage cumulé (fig. 7) ne tenant pas compte des animaux ne se déplaçant pas montre que l'évolution de la latence est en fait 

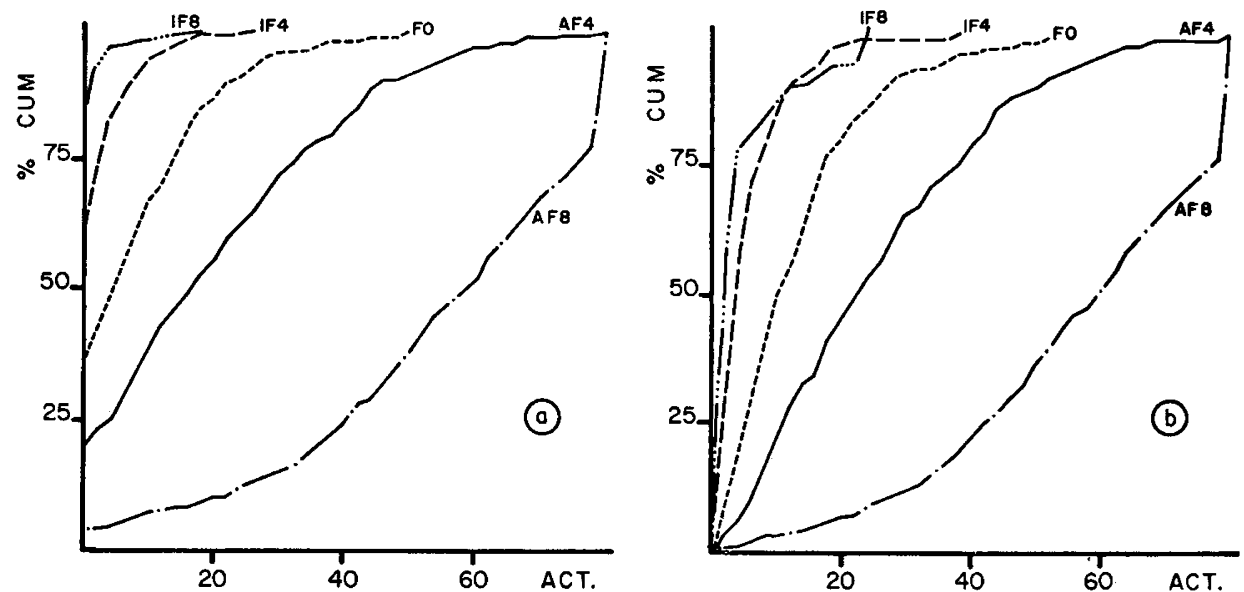

FIG. 5

Distribution en pourcentage cumulé de l'activité en tenant compte des animaux ne se déplaçant pas $(5 a)$ et sans en tenir compte $(5 b)$ à la $F 0, F 4$ et $F 8$.

Cumulative percent distribution of activity either with inactive animals taken into account (a) or disregarding inactive animals (b) in the starting pool,

F - F 0. the forth and the eighth generation.

- - Inactifs F 4.

Inactive strain, forth generation.

$-\cdots-$ Inactifs F 8 .

Inactive strain, eighth generation.

Actifs F 4.

Active strain, forth generation.

- - - - Actifs F 8.

Active strain, eighth generation.
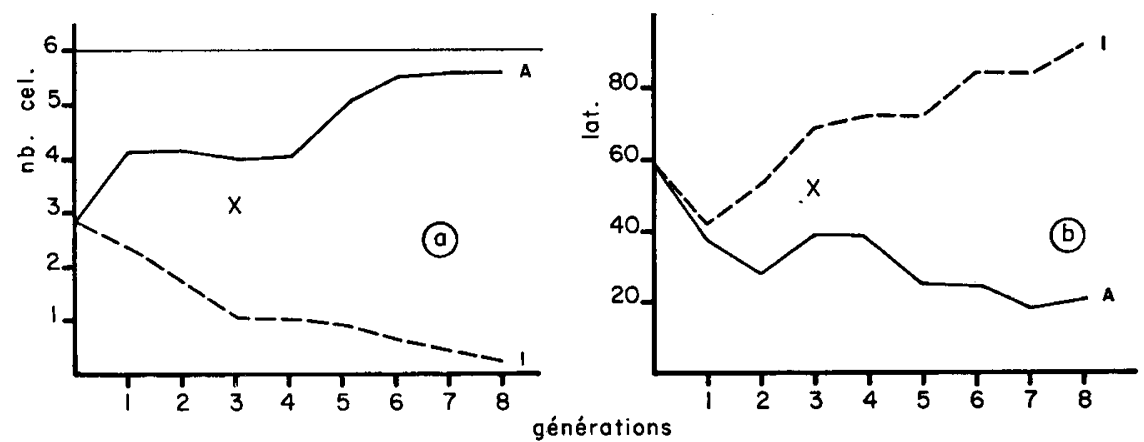

FIG. 6

Evolution, au cours de la sélection, du nombre de cellules utilisées (6 a) et de la latence (6 b) (idem figure 4).

Changes in the characters as a function of the generation for the number of cells (a) and the latency $(b)$ (see figure 4 for symbols). 
complexe. De la F 0 à la F 4, l'évolution de la latence n'est due qu'à l'évolution du pourcentage d'animaux ne se déplaçant pas. Par contre, de la $4^{\mathbf{e}}$ à la $8^{\mathrm{e}}$ génération, il y a aussi évolution de la moyenne de la latence pour les animaux qui se déplacent.

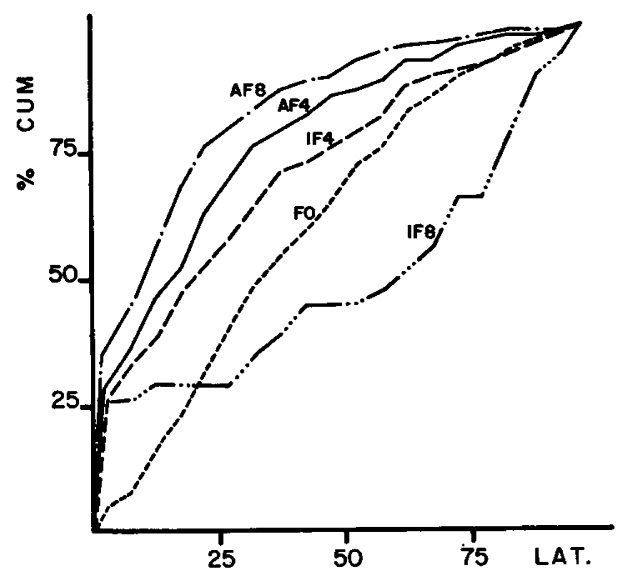

Fig. 7

Distribution en pourcentage cumulé, sans tenir compte des animaux ne se déplaçant pas, des latences à la F0, F4 et F8 (idem figure 5).

Cumulative percent distribution of the latencie disregarding inactive animals (see figure 5 for symbols).

De plus, dans les deux lignées, il y a eu une augmentation de la proportion des latences courtes (inférieures à 5 secondes) mais il s'agit de stratégies différentes. Dans la lignée active, ce sont réellement des latences courtes suivies d'une activité importante alors que dans la lignée inactive, il s'agit de course panique suivie d'immobilité.

De la $\mathrm{F} 0$ à la $\mathrm{F} 4$ et de la $\mathrm{F} 4$ à la $\mathrm{F} 8$, il y a donc une évolution globale à peu près régulière de l'activité et de la latence; cependant, le mécanisme de l'évolution a varié entre ces deux périodes. Alors que de la $F 0$ à la $F 4$, l'évolution globale est due à la fois à l'évolution de la moyenne et au pourcentage des animaux qui se déplacent de la $\mathrm{F} 4$ à la $\mathrm{F} 8$, seul l'un de ces deux critères varie pour l'activité dans la lignée inactive et pour la latence dans la lignée active. Il semble donc que, alors que le critère de sélection reste le même, le caractère sélectionné ait changé entre les deux périodes.

La fréquence des déjections (fig. 8 b) n'a pratiquement pas varié dans la lignée active. Par contre, pour les cris (fig. 8 a), les déjections dans la lignée inactive et les sauts (fig. $8 \mathrm{c}$ ), il y a eu une évolution conforme à ce que laissaient prévoir les corrélations, c'est-à-dire une augmentation de leur fréquence dans la lignée active et une diminution dans la lignée inactive.

Le tableau 2 donne les valeurs d'héritabilité réalisée suivies de leur intervalle de confiance et pour les héritabilités calculées les moyennes non pon- 
dérées des 8 générations et entre parenthèses des 4 premières générations pour la lignée inactive.

On constate que les héritabilités réalisées dans les deux lignées ne présentent des différences significatives que pour la latence et pour la fréquence des déjections.

On peut observer aussi que les valeurs d'héritabilité calculée, sont systématiquement inférieures aux valeurs d'héritabilité réalisées, ceci peut être dû à deux causes :

- une sous-estimation des héritabilités calculées due à la non-normalité des données qui fait que, même après transformation, on obtient des valeurs biaisées;

- une surestimation des valeurs d'héritabilité réalisées en raison de la sélection qui ne tient pas compte seulement du phénotype mais aussi de la moyenne de la frâtrie.
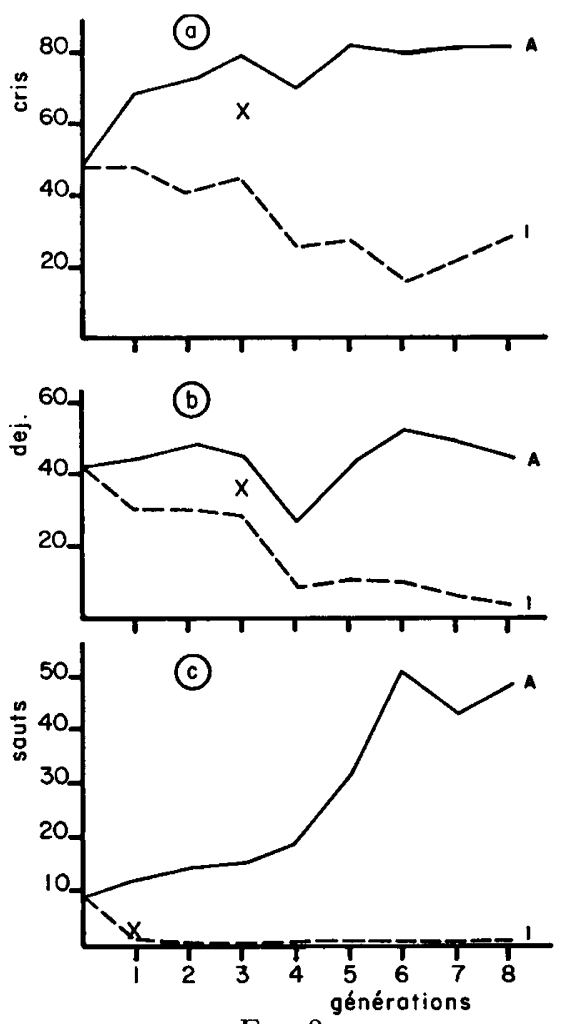

Fig. 8

Evolution, au cours de la sélection,

du pourcentage d'animaux émettant le cri de détresse pendant plus de $80 \mathrm{p} .100$ du test (8 a), du pourcentage d'animaux déféquant pendant le test $(8 \mathrm{~b})$

et du pourcentage d'animaux sautant pendant le test $(8 \mathrm{c})$ (idem figure 4).

Changes in the characters as a function of the generation for the percentage of birds emetting the distres call for more than 80 p. 100 of the duration of the test (a), the percentage of birds defecating during the test $(b)$

and the percentage of birds jumping during the test (c) (see figure 4 for symbols). 


\section{TABleau 2}

Valeurs des héritabilités réalisées ( \pm l'intervalle de confiance) et calculées.

Values of realized heritabilities ( \pm standard error) and calculated heritabilities.

\begin{tabular}{|c|c|c|c|c|}
\hline & \multicolumn{2}{|c|}{$\mathrm{H}^{2}$ réalisées } & \multicolumn{2}{|c|}{$\mathbf{H}^{2}$ calculées } \\
\hline & A & I & A & I \\
\hline Latence $\ldots \ldots \ldots \ldots$ & $0,46 \pm 0,05$ & $0,18 \pm 0,03$ & 0,15 & $0,11 \quad(0,17)$ \\
\hline Produit & $0,36 \pm 0,02$ & $0,38 \pm 0,01$ & 0,20 & $0,11 \quad(0,17)$ \\
\hline Activité & $0,39 \pm 0,03$ & $0,32 \pm 0,02$ & 0,19 & $0,11(0,17)$ \\
\hline Nombre de cellules .. & $0,23 \pm 0,03$ & $0,25 \pm 0,01$ & 0,10 & $0,08(0,12)$ \\
\hline Cris .... & $0,20 \pm 0,03$ & $0,23 \pm 0,06$ & 0,17 & $0,15(0,18)$ \\
\hline Déjections $\ldots \ldots \ldots$ & $0,04 \pm 0,06$ & $0,49 \pm 0,05$ & 0,10 & $0,06(0,10)$ \\
\hline Sauts & $0,31 \pm 0,03$ & $x$ & 0,16 & $x$ \\
\hline
\end{tabular}

Dans la colonne des héritabilités réalisées, le signe $\longleftrightarrow$ indique que les valeurs sont significativement différentes au seuil 0,05 . Les chiffres entre parenthèses après la valeur moyenne des héritabilités dans la lignée inactive représentent la moyenne des quatre premières générations.

The sign $\longleftrightarrow$ indicates that the values of the realized heritabilities are significant at the 0.05 level. For the inactive line the value in parentheses is the mean for the four first generations.

\section{TABLEAU 3}

Résultats synthétiques des croisements.

Global results of the cross.

\begin{tabular}{|c|c|c|c|}
\hline & Heterosis & $\begin{array}{c}\text { Différences } \\
\text { entre F.1 récipr. }\end{array}$ & $\begin{array}{l}\text { Influence } \\
\text { âge de la mère } \\
\text { (back-cross) }\end{array}$ \\
\hline Latence $\ldots .$. & 0 & $\mathrm{~J} 1$ et $2(\mathrm{IA}>\mathrm{AI})$ & 0 \\
\hline Activité & 0 & J $2(\mathrm{AI}>\mathrm{IA})$ & J 2 (jeune > âgée) \\
\hline Nombre de cellules & 0 & 0 & 0 \\
\hline Latence cris $\ldots \ldots$ & 0 & $\mathrm{~J} 1(\mathrm{IA}>\mathrm{AI})$ & $\begin{array}{c}\mathrm{BC} / \mathrm{I} \quad \mathrm{J} 1 \text { à } \mathrm{J} 4 \\
\text { (âgée }>\text { jeune) }\end{array}$ \\
\hline Déjections & $\mathrm{J} 2$ et $\mathrm{J} 3$ & 0 & 0 \\
\hline Sauts .. & $\begin{array}{ll} & \begin{array}{l}\text { Faible } \\
\text { J } 2\end{array} \\
\text { BC } / \mathbf{A}>\mathbf{A}\end{array}$ & J $4(\mathrm{IA}>\mathrm{AI})$ & 0 \\
\hline
\end{tabular}

Le signe $>$ indique une différence significative au seuil 0,05 .

The sign $>$ indicates that the differences are significant at the 0.05 level. 
On constate aussi que dans la lignée inactive, la moyenne des héritabilités calculée sur les quatre premières générations est toujours supérieure à celle calculée sur les huit premières générations. Ceci est probablement dû à ce que dès la quatrième génération, la plupart des animaux ont une activité et une fréquence des cris, des déjections et des sauts qui est nulle et une latence de 100 secondes.

\section{b) Croisements}

L'essentiel des résultats est résumé dans le tableau 3.

- On constate qu'il n'y a d'hétérosis au sens fort (F 1 non compris entre les valeurs de leurs parents) que pour les défécations à $\mathrm{J} 2$ et $\mathbf{J} 3$.

- Qu'il y a pour de nombreux caractères (latence à $\mathrm{J} 1$ et $\mathrm{J} 2$, activité à $\mathrm{J} 2$, latence des cris à $\mathbf{J}$ 1) un effet maternel significatif, allant dans le sens d'une dissemblance entre la mère et le poussin et dans le sens d'une ressemblance pour les sauts à $\mathrm{J} 4$.

- Que les poussins issus de mère jeune présentent certains caractères qui les rapprochent des animaux actifs. Ces animaux étant plus légers, l'accroissement de l'activité que l'on constate dans les tests d'habituation (FAURE, 1975, 1979 a ; JONES, 1977) ne peut pas être dû à la croissance.

\section{Conclusions}

Les valeurs d'héritabilité moyennes, l'absence presque complète d'hétérosis, de plateau de sélection, d'homéostase de développement ou d'assymétrie dans l'évolution des lignées divergentes montrent que le caractère mesuré par le test d'open-field n'a pas été soumis à une sélection naturelle directionnelle (BRUELL, 1967). De plus, la présence d'un effet maternel allant dans le sens d'une dissemblance entre la mère et ses descendants plaide en faveur d'une sélection naturelle stabilisante plutôt que d'une indifférence du caractère vis-à-vis de la sélection naturelle.

\section{3. - Caractères évoluant corrélativement à la sélection}

\section{a) Réactivité émotionnelle et taux de corticostérone}

La réactivité émotionnelle est le facteur le plus souvent invoqué pour expliquer le comportement en open-field des rongeurs. Nous avons donc testé cette hypothèse chez le poussin en utilisant des tests éthologiques, la mesure du rythme cardiaque et le taux de corticostérone.

Dans les souches non sélectionnées ou dans la lignée inactive, l'habituation entraîne une évolution vers le type actif (FAURE, 1975, 1979 a, 1980 b). Ce type doit donc être le moins émotif.

Les animaux de la lignée active présentent une immobilité tonique courte et une faible réactivité à une stimulation nouvelle (FAURE, 1975) ce qui confirme leur faible émotivité. 
Cependant, même si ces tests sont généralement acceptés comme des mesures de l'émotivité, l'interprétation des résultats peut se faire aussi bien en terme d'activité générale qu'en terme d'émotivité.

Pour déterminer laquelle de ces deux explications est la plus probable, nous avons soumis des animaux des deux lignées à un test de consommation alimentaire dans une mangeoire nouvelle. On constate dans ces conditions que la consommation des animaux de la lignée active est rapidement proche de la normale alors que même après 4 jours, celle des inactifs reste inférieure à la normale (FAURE, $1980 \mathrm{a}$ ). La différence entre les deux lignées est donc bien due à leur émotivité.

Les deux lignées ne présentent pas par contre de différences pour leur réactivité cardiaque mais diffèrent pour leur taux de corticostérone, plus élevé dans la lignée inactive (FAURE, 1980 a). Cette différence est augmentée par un agent stressant.

\section{b) Influences sociales sur le comportement en open-field}

Les animaux sont placés dans un open-field carré de $1 \mathrm{~m}$ de côté, qui permet de mesurer automatiquement l'activité d'un animal isolé mais aussi d'un animal à l'intérieur d'un groupe grâce au courant induit dans un conducteur par un petit aimant fixé à la patte du poussin (BESSEI \& BESSEI, 1974).

Dans ces conditions, on constate (fig. 9) qu'en test de groupe, l'activité des animaux de la lignée active est très fortement réduite alors que dans la lignée inactive la différence entre les deux conditions n'est pas significative (BESSEI, 1980).

Ce résultat montre que dans la lignée active, l'essentiel de l'activité est motivée par la recherche de partenaires sociaux alors que dans la lignée inactive cette motivation est faible ou ne s'exprime pas.

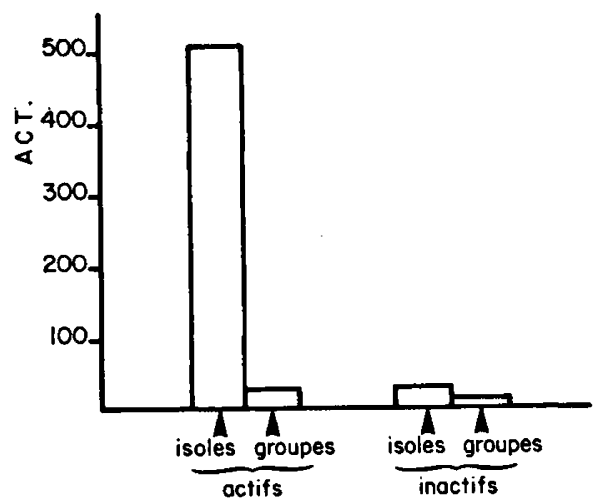

FIG. 9

Activité en open-field d'animaux testés isolés ou en groupe à l'âge de 3 jours pour les deux lignées.

L'activité est mesurée par le nombre de carrés de $10 \mathrm{~cm}$ de côtés visités en 4 minutes (d'après BESSEI, 1980).

Open-field activity of the two strains of birds tested isolated or in groups at 3 days of age. Activity in measured by the number of squares $(10 \times 10 \mathrm{~cm})$ entered in 4 minutes (from BESSEI, 1980). 


\section{c) Comportement social}

Le comportement social des animaux des deux lignées a été étudié dans trois conditions en raison de l'influence possible du milieu sur le comportement.

- Rencontres par paire en terrain neutre d'animaux ne se connaissant pas. Les rencontres ont été faites entre animaux de même lignée et entre des animaux des deux lignées (FAURE, 1977 a).

- Petits groupe d'une douzaine d'animaux les actifs et inactifs étant mêlés. Les animaux sont observés après 15 heures de privation d’aliment dans des conditions de compétition alimentaire telles qu'un seul animal peut manger à la fois (FAURE, 1977 b).

- Grands groupes d'une centaine d'animaux, les lignées étant séparées.

Ces trois méthodes représentent dans cet ordre une décroissance de l'information que l'on peut recueillir mais sont en même temps de plus en plus proches des conditions normales d'élevage.

Nous utiliserons «aptitude à la dominance» comme synonyme de probabilité qu'a un animal de gagner une rencontre et «agressivité » comme synonyme de probabilité qu'a un animal dominant de donner des coups.

On constate pour l'agressivité que, dans les conditions de rencontres par paire, les actifs sont les plus agressifs alors que le résultat inverse est obtenu dans les groupes constitués dès la naissance et mesurés dans leurs conditions habituelles d'élevage.

Nous pensons que cette différence entre les deux résultats est due à la néophobie des inactifs et que, dans les conditions de rencontre par paire, leur agressivité est inhibée par la nouveauté de la situation, alors que dans les conditions normales de vie (groupes constitués dès la naissance et placés dans un milieu connu) l'agressivité des animaux inactifs s'exprime normalement.

L'aptitude à la dominance (fig. 10) présente une relation complexe avec l'activité en open-field (FAURE, 1977 a). On constate qu'une activité en open-field moyenne correspond à une aptitude à la dominance élevée alors qu'une activité en open-field faible ou forte entraîne une faible aptitude à la dominance.

Une relation de même type avait été trouvée par CaNDLAND et al. (1970) entre aptitude à la dominance et rythme cardiaque chez le coq, cependant dans cette expérience la mesure du rythme cardiaque précédait immédiatement la rencontre par paire alors que dans la présente expérience la mesure du comportement en open-field est effectuée à l'âge de 2 ou 3 jours et les rencontres par paire à l'âge de 20 semaines. logiques.

Ce résultat peut être interprété en termes de génétique ou en termes étho-physio-

Sur le plan génétique, on a montré que l'aptitude à la dominance était très sensible à l'hétérosis (FAURE, 1977 c). L'hétérosis au niveau des gènes déterminant l'activité en open-field peut donc avoir un effet pléiotropique sur l'aptitude à la dominance. 


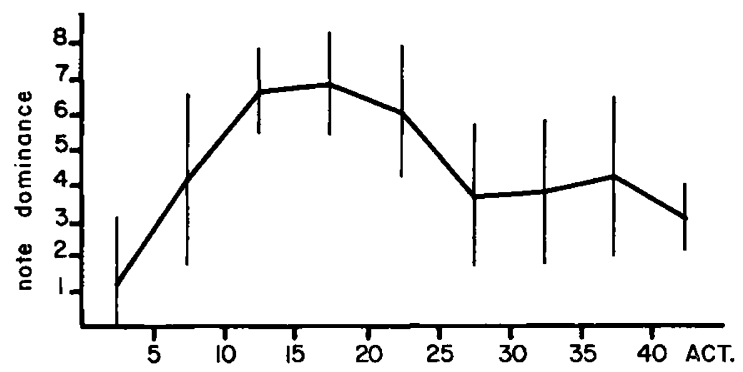

FIG. 10

Aptitude à la dominance

(nombre moyen de rencontres par paires gagnées à l'âge de 20 semaines) en fonction de l'activité en open-field (à l'âge de 2 ou 3 jours).

Les traits verticaux représentent l'intervalle de confiance à la moyenne.

\section{Dominance ability}

(at the age of 20 weeks) as a function of open-field activity (at 2 or 3 days of age).

Vertical lines show the standard error.

Sur le plan étho-physiologique un animal très émotif peut réagir à la mise en présence d'un congénère inconnu par des réactions de fuite et donc perdre la rencontre alors qu'un animal très peu émotif peut ne pas être préparé au combat (sécrétion d'adrénaline, augmentation du rythme cardiaque et de la glycémie...) et donc le perdre aussi.

\section{d) Taux des médiateurs du système nerveux central}

Ce travail a été réalisé en collaboration avec le laboratoire de Neurochimie de Strasbourg.

Nous avons pu montrer qu'après 3 générations de sélection, il y avait des différences significatives entre les deux lignées en particulier pour le taux de sérotonine qui est supérieur dans la lignée inactive aux âges de 15 et 28 jours dans toutes les zones de l'encéphale qui ont été étudiées.

Il n'y a par contre pas de différences aux âges plus précoces ce qui montre que les tests éthologiques sont plus discriminants que les mesures biochimiques.

\section{e) Caractères d'intérêt zootechnique}

Nous avons vu d'une part que nos lignées différaient par leur agressivité, or, l'agressivité est génératrice de stress (Mac BRIDE, 1958 a et b ; Williams et al., 1977). On sait, d'autre part, que les stress agissent sur la vitesse de croissance, la ponte et la viabilité (Graig \& ToTH, 1970; BISWAS \& CRAIG, 1971; Gover et al., 1972; Brown \& Nestor, 1973, 1974 ; Gross \& Siegel, 1973 ; Siegel, 1978). Il était donc important d'un point de vue pratique de voir comment ces différents caractères évoluent au cours de la sélection. 


\section{- Conformation}

Nous avons observé que les animaux de la lignée inactive sont plus lourds que ceux de la lignée active (fig. 11). Ces différences disparaissent cependant à la huitième génération. On observe des évolutions comparables pour la plupart des autres caractères de conformation : angle de poitrine, diamètre du tarse, poids adulte (FAURE, 1977 d).

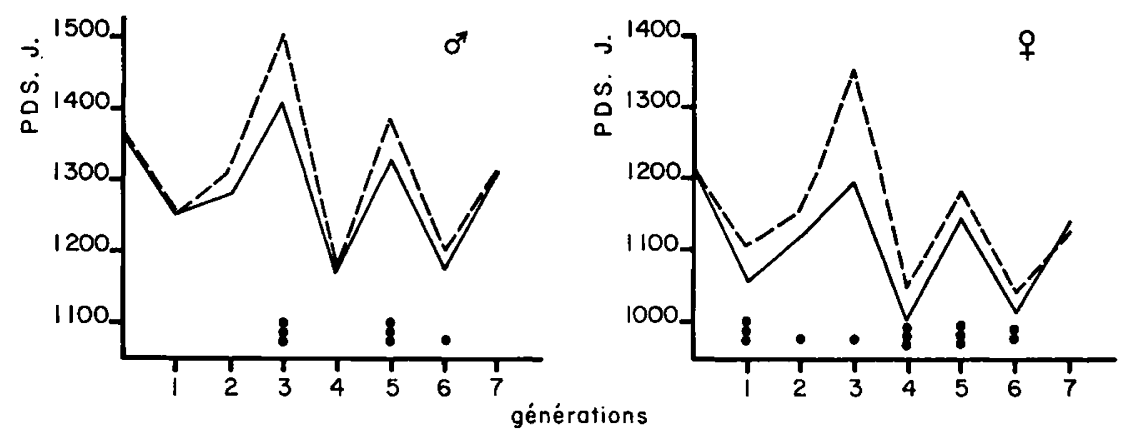

Flu. 11

Evolution du poids à 6 semaines (en g) au cours de la sélection pour les deux sexes.

Changes in body weight (in grams) for males (a) and females (b) as a function

$\bullet=\mathrm{p}<0,05$.

$\cdots=\mathrm{p}<0,01$

$\bullet \bullet=\mathrm{p}<0,001$.

of the generation.

Ce résultat est très probablement dû à une dérive génétique puisque l'on n'observe pas de différence pour le comportement en open-field d'animaux sélectionnés pour la forme de leur courbe de croissance (RICARD, 1975 ; FaURE \& RICARD, 1977).

\section{- Reproduction}

La plupart des critères de reproduction ne sont que peu ou pas différents entre les deux lignées ou dans le cas où les différences sont significatives, elles s'inversent ou s'annulent souvent d'une génération à l'autre. Les seuls caractères constants sont la précocité sexuelle, plus grande dans la lignée inactive et le nombre de poussins par poule et par jour qui est plus élevé dans la lignée active.

\section{- Résistance aux agents pathogènes}

Les résultats d'épreuve directe de résistance aux maladies montrent un avantage de la lignée inactive pour la résistance à la maladie de Marek et à la coccidiose (Eimeria tenella) (FAURE \& YVORE, 1980). Il n'y a par contre pas de différences entre les lignées pour la résistance à Escherichia coli ou Salmonella pullorum.

On constate par contre que les deux lignées ont une dynamique du taux d'anticorps différente. Dans la lignée active, on a une croissance rapide de ce taux suivi d'une décroissance. Au contraire, la lignée inactive présente une croissance plus lente mais le taux reste ensuite stable. 


\section{Conclusions}

Nous avons vu que pour l'ensemble des caractères d'intérêt zootechnique il n'y avait que peu ou pas de différences entre les deux lignées. Il faut cependant remarquer que nos conditions expérimentales étaient généralement peu stressantes (faibles densités). Dans des conditions plus stressantes, il est probable que nous aurions observé un avantage pour les animaux de la lignée active moins sensibles aux stress.

\section{Discussion générale}

Le test d'open-field est souvent choisi aussi bien chez les rongeurs que chez les oiseaux en raison de ses caractéristiques méthodologiques (rapidité, standardisation). Afin de l'appliquer à notre animal expérimental, il nous est apparu nécessaire d'en faire une analyse critique.

Ce test a surtout été utilisé pour les rongeurs et son application au poulet est récente (BoyEZ et al., 1970) et les motivations invoquées sont généralement le comportement de défense, l'exploration ou le marquage de territoire. Il nous est apparu nécessaire de comparer ces trois types de comportement au comportement en openfield du poussin.

Les résultats de cette comparaison sont regroupés dans le tableau 4. On constate que le comportement territorial peut être éliminé d'emblée chez le poussin puisqu'il est absent à l'âge où le test est effectué.

\section{TABLEAU 4}

Principales caractéristiques des comportements territoriaux, exploratoires, face à un danger et du comportement en open-field.

Main characteristics of territorial behaviour, exploration, fear reactions and open-field behaviour.

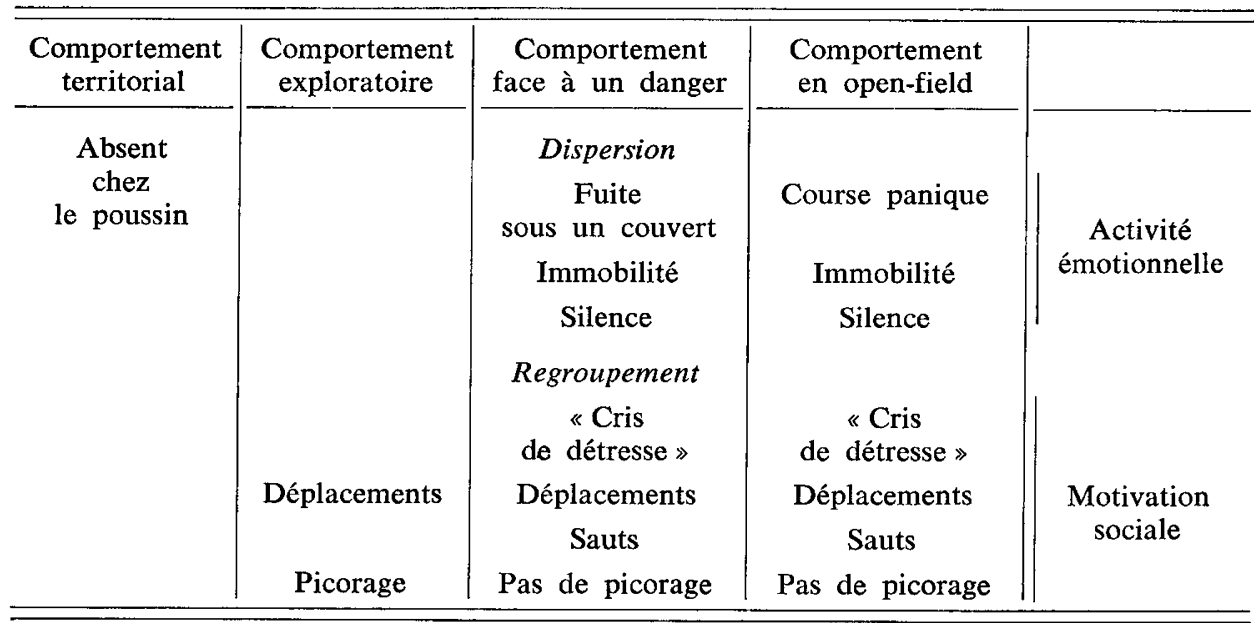


Le comportement exploratoire est caractérisé chez le poussin par des déplacements mais aussi par des picorages de l'environnement qui sont pratiquement absents dans le comportement en open-field.

Le comportement face à un danger est beaucoup plus comparable au comportement en open-field. Il est caractérisé par une phase de dispersion comprenant la fuite vers un couvert, suivie d'une période d'immobilité et de silence et par une phase de regroupement de la couvée caractérisée par des cris de détresse, des déplacements et des sauts (KRUIJT, 1964).

Cette séquence de comportements est remarquablement parallèle à ce que l'on observe en open-field où une courte phase de fuite rapide (souvent absente) peut correspondre à la recherche d'un couvert (absent aussi) et est suivie d'une période d'immobilité et de silence puis d'une période active avec émission des cris de détresse.

Si ce parallèle entre comportement en open-field et comportement face à un danger n'est pas une coïncidence, on doit s'attendre à ce que les réactions de la première phase soient dues à l'émotivité alors que celle de la seconde phase doivent avoir une motivation sociale.

A partir de ces conclusions, nous pouvons essayer de représenter l'intensité ou la fréquence des différents comportements en fonction de l'activation émotionnelle (fig. 12).

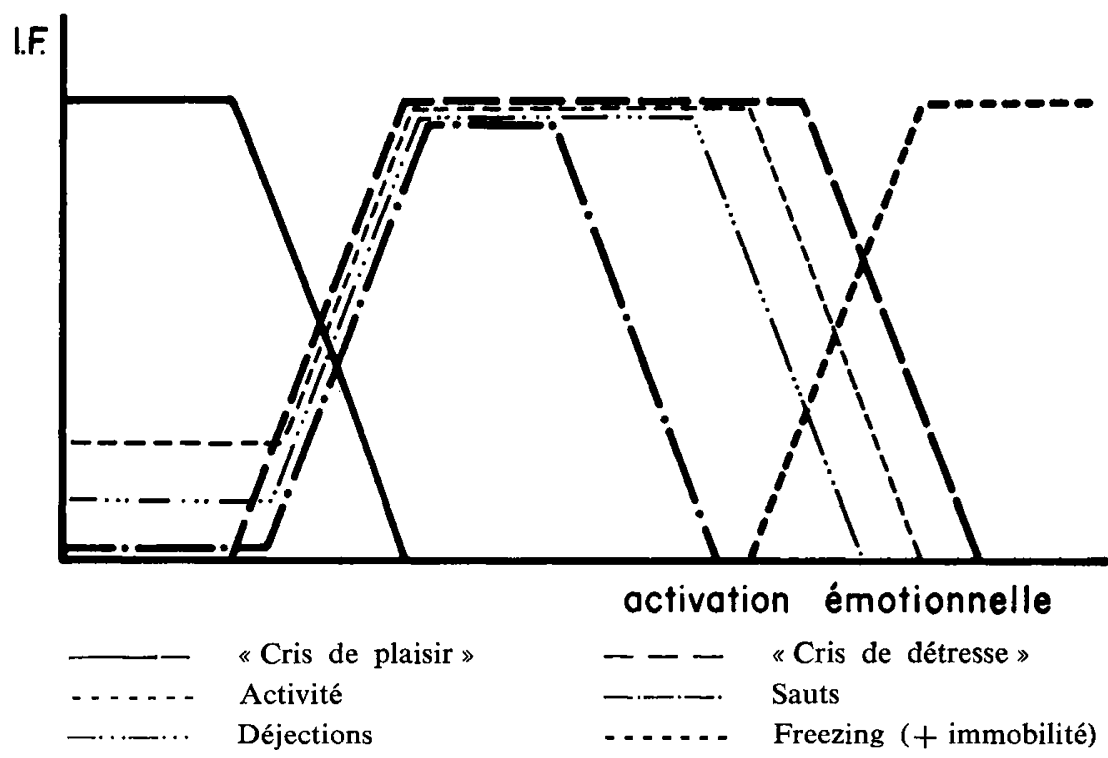

FIG. 12

Evolution de l'intensité (I) ou de la fréquence $(F)$ des comportements en open-field en fonction de l'activation émotionnelle.

Intensities or frequencies of certain behaviour patterns at varying levels of fear induction. 
Nous avons sur la gauche de la figure une zone d'activation émotionnelle très faible qui correspond à ce que l'on observe pour l'animal placé dans les conditions normales d'élevage.

Au centre de la figure, il y a une zone d'activation émotionnelle modérée qui correspond au comportement en open-field des actifs ou des inactifs habitués durant laquelle les déplacements, les cris, les sauts et les défécations sont fréquents et, enfin, une troisième zone où ces comportements sont inhibés par l'activation émotionnelle et remplacés par de l'immobilité ou du freezing. Entre ces trois zones se trouvent deux zones de transition, dont l'une à droite a été très étudiée (comportement en début de test d'open-field) alors que l'autre est moins bien connue.

Ce modèle s'il explique correctement la disparition des comportements de type actif par leur inhibition lorsque l'activation émotionnelle est élevée n'explique par contre pas du tout leur augmentation lorsque l'on passe de la phase 1 à la phase 2 (partie gauche de la fig. 12).

Nous avons vu dans la description de la réaction normale des poussins face à un danger que la deuxième partie de la réaction (caractérisée par des comportements de type actif) devait avoir une motivation sociale puisqu'elle entraîne le regroupement de la couvée.

De plus, les résultats expérimentaux disponibles montrent que dans les tests de groupe, l'activité est réduite, comme elle est réduite dans le cas d'animaux élevés isolés et testés isolés (Gallup \& SuAREZ, 1980).

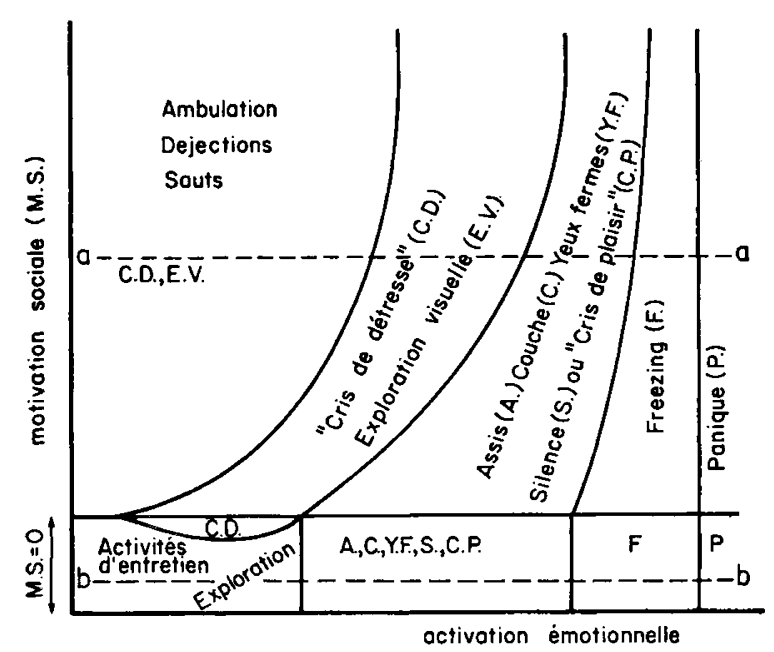

FIG. 13

Essai de représentation des différents comportements en open-field en fonction de la motivation sociale et de l'activation émotionnelle.

Hypothetical diagram representing various behaviour patterns as a function of social motivation and fear induction. 
Nous pouvons donc proposer un modèle à deux dimensions (FAURE et al., 1982) (fig. 13) tenant compte à la fois de l'activation émotionnelle et de la motivation sociale. Dans le modèle, la ligne «a » correspond aux conditions habituelles des tests d'openfield, c'est-à-dire des animaux élevés en groupe et testés isolés et la ligne «b 》 aux animaux testés dans les conditions sociales d'élevage.

Pour la ligne « $a »$, la motivation sociale est forte et au début du test l'activation émotionnelle est forte aussi, la séquence de comportement suivie est donc : course panique et freezing éventuels, immobilité et silence, puis cris de détresse et exploration visuelle et enfin, ambulation, défécation, sauts avec toujours exploration visuelle et cris de détresse.

La ligne « $b$ » correspond à une motivation sociale nulle et à une activation émotionnelle forte en début de test. La séquence de comportements est alors course panique et freezing éventuels, immobilité et silence puis exploration et activités d'entretien.

Ce modèle est à l'évidence en partie conjectural car si les deux lignes « a » et «b» correspondent à des situations bien étudiées, la partie située entre les deux lignes demanderait des recherches complémentaires pour que soit précisée l'interaction entre émotivité et motivation sociale.

Le modèle proposé montre que la divergence des deux lignées a pu être due à une sélection de la motivation sociale, une sélection de l'émotivité ou une combinaison des deux.

Cette dernière hypothèse est la plus probable puisque un test d'émotivité : le test d'immobilité tonique permet de mettre en évidence des différences entre les deux lignées dès la deuxième génération de sélection mais qu'on observe aussi une très forte influence des conditions sociales de test dans la lignée active alors que cette influence est faible dans la lignée inactive.

Il est donc probable que la lignée active peut se caractériser à la fois par une faible émotivité et une forte motivation sociale alors que la lignée inactive présente les caractéristiques inverses.

Le même type de sélection a probablement aussi été réalisé tout au long du processus de domestication. En effet, dans les caractères classés par Hall (1969) comme favorables à la domestication, on trouve entre autre la vie en groupes sociaux de grande taille et une réactivité faible à des changements brusques de l'environnement.

Cette conclusion est confirmée par les expériences récentes de domestication (Belyaev, 1979 ; Belyaev \& Ternovskaya, 1973) ou par la comparaison des espèces domestiques et sauvages (Barnett, 1958; Galef \& Clark, 1971 ; Poole, 1972 ; LeOPOLD, 1944 ; Desforges \& Wood-Gush, 1975 a et b). Dans tous les cas où ce travail a été fait on constate une réduction de l'émotivité et une augmentation de la sociabilité dans l'espèce domestique. Cette sélection n'a cependant pas dû être très efficace puisqu'on ne constate pas d'assymétrie dans les réponses des deux lignées à la sélection pour le comportement en open-field.

La sélection pour une activité forte pourrait permettre d'augmenter les caractéristiques favorables à la domestication et donc permettre d'avoir des animaux plus adaptables aux conditions de l'élevage intensif. Or, l'adaptabilité semble être le carac- 
tère le plus important à sélectionner car, de tous les caractères de comportement, il est le seul à être manifesté quelles que soient les conditions d'élevage.

Reçu pour publication le 31 mars 1982.

\section{Summary \\ Genetic analysis of open field behaviour of the young chick \\ (Gallus gallus domesticus)}

The open-field test has been used quite often on laboratory rodents but a lot less often on birds. It has the advantage of being very standardized and quick and so is useful for genetic studies.

\section{I. - Study of open-field behaviour} activity.

The open-field used allows the automatic recording of the animals's latency and

A circular enclosure measuring $1 \mathrm{~m}$ in diameter and divided into 6 sections by 6 light beams each ending in a photo cell was used. The cries, defecations and jumps were measured by the observer.

A sample of several strains was examined and we were able to show that the different measurements were correlated (negative correlations between the latency and the other characters and positive between all the other characters).

\section{II. - Genetic study}

After eight generations of selection, the lines are very different and the distributions practically do not overlap. The heritabilities of the different characters show low or medium values. The characters studied do not show heterosis or developmental homeostasis. We established, for several characters, the presence of a maternel effect, tending towards a dissimilarity between the dam and her descendants.

This group of characters allows the deduction to be made that the motivation underlying the behaviour in open-field is subjected to a natural stabilizing selection.

\section{III. - Characters evolving correlatively with the selection}

The animals of the active line are characterized by a lower emotionality and a reduced corticosterone level. They are also less aggressive. The animals with medium open-field activity have a higher dominance ability.

In contrast, the lines show few differences on the zootechnical side.

A model is given to try to explain the open-field behaviour of the young chick with relation to emotionality and social motivation.

The active animals, not very emotional, aggressive or sensitive to stress, seem to correspond to an animal which is more adaptable to modern rearing conditions and this type of selection, easily usable on great numbers of animals, could be used in commercial selection.

\section{Références bibliographiques}

BARNETT S.A., 1958. Experiments in «neophobia» in wild and laboratory rats. Br. J. Psychol., 49, 195-201.

Belyaev D.K., 1979. Destabilizing selection as a factor in domestication. J. Hered.. 70, 301-306. 
Belyaev D.K., Ternovskaya Y.G., 1973. Behaviour and reproductive functions of animals. Genetica, 9, 53-63.

BEsSEI W., 1980. Essential behavioural needs : the mixed motivation approach. In : R. Moss ed., The laying hen and its environment, 167-174, Martinus Nijhoff Publishers, The Hague.

Besser H., Bessei W., 1974. Eine Messalange zum Überwachen der lokimotorischen Activität von Huhnern in Bodenhaltung. Arch. Geflüg., 38, 94-99.

Brswas D.K., Craig J.V., 1971. Social tension indexes and egg production traits in chickens. Poult. Sci., 50, 1063-1065.

Boyer J.P., 1958. Théorie et calcul des indexes de sélection. Ann. Zootech., 7, 193-242.

Boyer J.P., Melin J.M., Ferre R., 1970. Différences génétiques de comportement exploratoire expérimental chez le poussin. Premiers résultats. XIV Cong. Mond. Avic., Madrid, 2, 21-25.

BRoadhuRST P.L., JinKs J.L., 1961. Biometrical genetics and behaviour : reanalysis of published data. Psychol. Bull., 58, 337-362.

Brown K.I., Nestor K.E., 1973. Some physiological responses of turkeys selected for high and low adrenal responses to cold stress. Poul. Sci., 52, 1948-1954.

Brown K.I., Nestor K.E., 1974. Effect of stress on rate of gain and feed conversion of ohio low and high adrenal response turkey lines. Ohio Agric. Dev. Center, Res. Summary, 80, 4-6.

BRuELl J.H., 1967. Behavioral heterosis. In : J. HiRCH ed., Behavior-genetic analysis, 270-286, Mac Graw-Hill, New York.

Candland D.K., Taylor D.B., Dresdale L., Leiphart J.M., Solov S.P., 1969. Heart rate, agression and dominance in the domestic chicken. J. comp. physiol. Psychol., 70, 417-423.

COCHez L.P., Pero L., 1954. Transformation des mesures brutes en écarts réduits pour atténuer l'incidence des variations de milieu. Xth World Poult. cong., Edinburgh, 16-19.

Craig J.V., Toth S., 1970. Influence de la sélection génétique pour la dominance sociale et la stabilité du troupeau sur la productivité des poules. Hongrois. Allatenyéstré Magyar, 19, 351-357.

DESFORges M.F., Wood-Gush D.G.M., 1975 a. A behavioural comparison of domestic and mallard ducks. Habituation and flight reactions. Anim. Behav., 23, 692-697.

Desforges M.F., Wood-Gush D.G.M., 1975 b. A behavioural comparison of domestic and mallard ducks. Spacial relationships in small flocks. Anim. Behav., 23, 698-705.

Falconer S., 1961. An introduction to quantitative genetics. Oliver and Boyd, Edinburgh, London.

FAURE J.M., 1975. Etudes des liaisons entre comportement en open-field et émotivité chez le jeune poussin. Ann. Génét. Sél. anim., 7, 197-204.

FAURE J.M., 1977 a. Relation entre l'activité en open-field et l'aptitude à la dominance chez le coq (Gallus gallus). Biol. Behav., 2, 193-201.

FAURE J.M., 1977 b. Relation entre la hiérarchie sociale et la priorité d'accès à la mangeoire chez Gallus gallus. Biol. Behav., 2, 329-336.

FAURE J.M., 1977 c. Effet d'hétérosis dans le comportement agonistique du coq. Biol. Behav., 2, 181-188.

FAURE J.M., 1977 d. Relation entre la croissance et l'activité en open-field chez la poule. I - Effet de la sélection pour l'activité en open-field sur la croissance en poids et la conformation. Ann. Génét. Sél. anim., 9, 241-245.

FAURE J.M., 1979 a. Effet de l'habituation sur le comportement en open-field du jeune poussin (Gallus domesticus). Biol. Behav., 4, 241-248.

FAuRE J.M., 1979 b. Influence de la souche et du sexe sur le comportement en open-field du jeune poussin. Biol. Behav., 3, 19-24.

FAURE J.M., 1980 a. To adapt the environment to the bird or the bird to the environment. In : R. Moss Ed., The laying hen and its environment, 19-30, Martinus Nijhoff Publishers, The Hague. 
FAURE J.M., 1980 b. Evolution du comportement au cours d'un test open-field long chez le poussin. Biol. Behav., 5, 29-35.

FAURE J.M., 1981 a. Behavioural measures for selection. In First European Symposium on poultry Welfare, Kege, 10-12 juin 1981, L.Y. SerEnSEN ed., 37-41.

FAURE J.M., 1981 b. Bidirectional selection for open-field activity in young chicks. Behav. Genet., 11, 135-143.

Faure J.M., Folmer J.C., 1975. Etude génétique de l'activité précoce en open-field du jeune poussin. Ann. Génét. Sél. anim., 7, 123-132.

FAURE J.M., JONES R.B., 1981 a. A comparison of automatic recording and direct observation of open-field behaviour in domestic chicks. I.R.C.S. Med. Sci., 9, 112-113.

FAuRe J.M., Jones R.B., Bessei W., 1982. Fear and social motivation as factors in openfield behaviour of the domestic chick : A theoretical consideration. Biol. Behav. (accepté pour publication).

FAURE J.M., RiCARD F.H., 1977. Relation entre la croissance et l'activité en open-field chez la poule. II - Effet de la sélection pour le poids vif sur l'activité en open-field. Ann. Génét. Sél. anim., 9, 247-250.

FAURE J.M., Yvore P., 1980. Influence d'une sélection sur le comportement sur la réponse à l'infection coccidienne chez la poule. Ann. Génét. Sél. anim., 12, 377-381.

Fuller J.L., Du Buis E.M., 1969. The behaviour of dogs. In : E.S.E. Habez Ed., The behaviour of domestic animals, Baillere, Tindall and Cox, London.

GaleF B.G., Clark M.M., 1971. Social factors in the poison avoidance and feeding behaviour of wild and domesticated rat pups. J. comp. physiol. Psychol., 75, 341-357.

GalluP G.G. Jr., Suarez S.D., 1980. An ethological analysis of open-field behaviour in chickens. Anim. Behav., 28, 368-378.

Gover R.M., Anderson D.L., Damon R.A., Ruggle L.H., 1972. The effect of bird density dietary energy, light intensity and cage level on the reproductive performance of heavy type chickens in wire cages. Poult. Sci., 51, 565-575.

Gross W.B., Siegel P.B., 1973. Effect of social stress and steroids on antibody production. Avian Diseases, 17, 807-815.

GuYomaRC'H J.C., 1975. Les cycles d'activité d'une couvée naturelle de poussins et leur coordination. Behaviour, 53, 31-75.

HALE E.B., 1969. Domestication and the evolution of behaviour. In : E.S.E. HAFEZ Ed., The behaviour of domestic animals, Baillere, Tindall and Cox, London, 22-44.

JONES R.B., 1977. Repeated exposure of the domestic chick to a novel environment. Effects on behavioural response. Behav. Process., 2, 163-173.

Jones R.B., FaURE J.M., 1981 a. Sex effect on open-field behaviour in the domestic chick as a function of age. Biol. Behav., 6, 265-272.

Jones R.B., FAURE J.M., 1982. Open-field behaviour of male and female domestic chicks as a function of housing, test situation and novelty. Biol. Behav., 7, 17-25.

KRUIJT J.P., 1964. Ontogeny of social behaviour in burmese red jungle fowl (Gallus gallus spadiceus), Bonaterre. Behaviour suppl., 12, 1-201.

LEOPOLD A.S., 1944. The nature of heritable wildness in turkeys. Condor, 46, 133-197.

MAC BRIDE G., 1958 a. Relationships between aggressiveness and egg production in the domestic fowl. Nature, 181, 858-859.

MAC BRIDE G., $1958 \mathrm{~b}$. The relationship between aggressiveness, peck order and some characters of selective significance in the domestic hen. Proc. r. phys. Soc. Edinb., 27, 56-60.

MATHER K., 1949. Biometrical genetics : the study of continous variation. London, Methuen.

Poole T.B., 1972. Some behavioural differences between the european polecat Mustela putorius, the feret Mustela furio, and their hybrids. J. Zool. Lond., 166, 25-35.

Ricard F.H., 1975. Essai de sélection sur la forme de la courbe de croissance chez le poulet. Ann. Génét. Sél. anim., 7, 427-443.

Siegel P.B., 1978. Some thoughts on poultry behaviour. Hohenheimer Arbeiten, 93, 25-34.

Williams C.G., Siegel P.B., Gross W.B., 1977. Social strife in cockerels flocks during the formation of peck right. Appl. anim. Ethol., 3, 35-45.

Wood-GusH D.G.M., 1959. A history of the domestic chicken form antiquity to the 19th Century. Poult. Sci., 38, 321-326. 